\title{
USE OF MONTE CARLO SIMULATIONS FOR THE CALCULATION OF THE UNCERTAINTY ESTIMATE IN THE ELECTROCHEMICAL DETECTION OF URIC ACID IN PLASMA SAMPLES
}

\author{
DADAMOS, Tony Rogério de Lima ${ }^{1 *}$; DAMACENO, Airton Juliano ${ }^{1}$ FERTONANI, Fernando \\ Luis ${ }^{1}$, BETTENCOURT SILVA, Ricardo Jorge Neves ${ }^{2}$
}

${ }^{1}$ Universidade Estadual Paulista, Campus de São José do Rio Preto Instituto de Biociências, Letras e Ciências Exatas R. Cristóvão Colombo, 2265 - Jardim Nazareth São José do Rio Preto - SP, Brasil

(fone: + 5517 3221-2200)
${ }^{2}$ Centro de Química Estrutura - Facudade de Ciências da Universidade de Lisboa, Campo Grande, 1749-016 Lisboa, Portugal, Fone +351962 517081 (fone +35121750 0000)
${ }^{*}$ Autor correspondente
e-mail: tonyrlrl@yahoo.com.br

Received 27 October 2017; accepted 14 November 2017

\section{RESUMO}

Este trabalho apresenta uma alternativa eletroquímica simples e confiável para a determinação de ácido úrico em soro humano em comparação com o método de referência colorimétrico enzimático. A qualidade das medições eletroquímicas foi avaliada comparando sua incerteza com um valor alvo de 0,56 mg $\mathrm{dL}^{-1}$ e as medições utilizou-se o método de adição padrão. $O$ eletrodo de trabalho modificado é composto por $25 \%$ de lignina, $60 \%$ de nanografite, $15 \%$ de óleo mineral e cobre metálico eletrodepositado. A incerteza de medição foi estimada pela abordagem "bottom-up", a partir da qual componentes de incerteza foram combinadas aplicando-se a lei de propagação de incerteza (LPI), o método numérico de Kragten e o Método de Simulações de Monte Carlo (MCM). O procedimento analítico foi aplicado com sucesso na análise de soros fisiológicos adicionados a 1,0, 3,0, 5,0, 7,0 e 9,0 $\mathrm{mg} \mathrm{dL}^{-1}$ de AU e para outras duas amostras de soro fisiológico humano. Os resultados das medições, realizadas para as diferentes amostras de soro, empregando-se o método proposto, associado aos diferentes métodos para a estimativa de cálculo da incerteza, apresentaram valores suficientemente baixos e metrologicamente equivalentes, no qual os resultados apresentaram-se metrologicamente compatíveis com os valores de referência estimados.

Palavras-chave: Incerteza de Medição, Análises Clínicas, Simulações de Monte Carlo.

\section{ABSTRACT}

This work presents a low cost, simple and adequately reliable electrochemical alternative for the determination of uric acid in human serum in comparison to the enzymatic colorimetric reference method. The quality of the electrochemical measurements was assessed by comparing its uncertainty with a target value of $0.56 \mathrm{mg} \mathrm{dL}^{-1}$ and the measurements were carried out by the standard addition method. The modified working electrode consists of $25 \%$ lignin, $60 \%$ nanocarbon, $15 \%$ mineral oil and electrodeposited metallic copper. The uncertainty of measurement was estimated by the bottom-up approach, from which uncertainty components were combined by applying the uncertainty propagation law (LPI), the numerical method of Kragten and the Monte Carlo Simulation Method (MCM). The analytical procedure was successfully applied in the analysis of physiological sera added at 1.0, 3.0, 5.0, 7.0 and $9.0 \mathrm{mg} \mathrm{dL}^{-1}$ of $A U$ and for two other samples of human saline. The results of the measurements carried out for the different serum samples using the proposed method, associated to the different methods for estimating the uncertainty, showed sufficiently low and metrologically equivalent values, in which the results were shown to be compatible with the estimated reference values.

Keywords: Measurement Uncertainty, Clinical Analyzes, Monte Carlo Simulations. 


\section{INTRODUÇÃO}

O ácido úrico $(\mathrm{AU})$ é um composto de grande interesse biomédico, pois ele executa funções essenciais no metabolismo dos mamíferos. O ácido úrico é o primeiro produto final do metabolismo das purinas. Níveis anormais de AU são indicativos de sintomas para várias doenças, dentre elas o infarto (Wu et al., 2006). O nível de concentração do AU em fluidos corporais tais como como células, plasma sanguíneo e urina é um biomarcador para muitas condições clínicas, incluindo hiperglicemia, gota e doença de Lesch-Nyhan (Rho et al, 2011). Numerosas doenças, condições fisiológicas, alterações bioquímicas, fatores sociais e ambientais estão associados a aumentos da concentração de ácido úrico plasmático (Moriwaki et al., 2014).

O procedimento padrão utilizado nos laboratórios de análises clínicas para a análise de $A U$ em soro sanguíneo é o uso do método colorimétrico enzimático, empregando-se a técnica espectrofotométrica, a partir do qual o ácido úrico é oxidado pela uricase (UOD), catalisada pela peroxidase (POD), produzindo um produto de cor vermelha. A absorbância do composto formado é medida em $505 \mathrm{~nm}$, sendo diretamente proporcional à concentração de ácido úrico da amostra (Kageyama, 1971). A desvantagem desse procedimento é o alto custo da análise, a interferência causada pela presença de fluoreto que inibe a ação da uricase; e a presença de turbidez em certas amostras. A presença de fluoreto e de turvamento na amostra conduzem a resultados de medição equivocadamente elevados (Benine et al., 2014).

Outras técnicas utilizadas na determinação de ácido úrico encontrados na literatura são a fluorimétrica (Jin et al., 2016), alternativas espectrofotométricas (Khajehsharifi et al. 2014), além das técnicas eletroquímicas como a voltametria de onda quadrada (Filik et al., 2014), pulso diferencial (Uzun et al., 2015) e a amperometria (Guo et al., 2016).

As técnicas eletroquímicas tem importante aplicabilidade para esta finalidade devido ao seu baixo custo, devido aos avanços da eletrônica, simplicidade de construção e de modificação de eletrodos, além de resultados excelentes obtidos para determinação de ácido úrico. Outro aspecto importante para o emprego de eletrodos modificados é que estes permitem uma maior interação entre as espécies presentes em solução e a superfície do eletrodo, assegurando maior seletividade e sensibilidade (Martin et al., 2012). Por estas razões, é bem visto o desenvolvimento de soluções de baixo custo como a utilização da técnica eletroquímica implementada sob métodos adequados.

Devido a sensibilidade da técnica eletroquímica aos efeitos da matriz analítica, utiliza-se o método de quantificação de adição de padrão (MAP). O MAP apresenta limitação em relação à quantidade de amostra necessária para proceder-se às adições de padrões, o que dificulta a sua aplicação. No entanto, a realização de adições cumulativas do padrão à uma quantidade fixa de amostra, torna mais viável a implementação do MAP, porém requer a ponderação da diluição progressiva da amostra (Gonçalves et al., 2016).

Assim, se a diluição progressiva da amostra produzir fatores de diluição equivalentes, as condições de matriz na calibração serão equivalentes, minimizando-se as interferências na análise desta natureza. A adição de padrão é especialmente apropriada quando a composição da matriz da amostra é desconhecida ou complexa, e afeta o sinal analítico. Logo, o método de adição padrão compensa uma série de interferências ao se adicionarem quantidades conhecidas de analito à amostra desconhecida (Steliopoulos, 2015).

Para que uma determinação analítica seja adequada, esta deverá apresentar uma incerteza conhecida inferior a um valor máximo admissível designado como incerteza alvo (Bettencourt da Silva, 2016). A incerteza de medição é definida como um parâmetro associado ao resultado de uma medição, que caracteriza a dispersão dos valores que podem ser razoavelmente atribuídos ao mensurando (VIM, 2012). Em se tratando de incerteza alvo, especificamente para o $\mathrm{AU}$, a quantidade de ácido úrico em indivíduos saudáveis, varia no intervalo de 2,0 a $8,0 \mathrm{mg} \mathrm{dL}$ 1 , e este intervalo pode ser utilizado para se definir a incerteza alvo, conforme o Guia Eurachem/CITAC (Eurachem, 2012).

É importante assegurar a qualidade das medições de $A U$, visto que valores superiores a $8,0 \mathrm{mg} \mathrm{dL}^{-1}$ podem servir de indicativo, de que o paciente seja portador de ao menos uma das possíveis doenças associadas a sua presença: 1- gota; 2- insuficiência renal; 3- leucemia; 3policitemia; 3- mieloma múltiplo; 4- intoxicação por chumbo; e 5- cetoacidose, na síndrome de Lesch-Nyhan e nas hiperuricemias idiopáticas. 
Por outro lado, valores inferiores a $2,0 \mathrm{mg} \mathrm{dL}^{-1}$ são pouco frequentes, mas podem indicar doenças tais como: 1- a síndrome de Fanconi; 2a doença de Wilson; e 3- doenças malignas, tais como linfoma de Hodgkin e carcinoma broncogênico, obviamente após avaliação prévia do quadro clínico do paciente (Zuo et al., 2016).

Para a avaliação da incerteza e para a garantia da qualidade dos resultados analíticos das medições são apresentadas na literatura várias abordagens para a avaliação da incerteza; podendo ser destacada a abordagem "bottomup", a qual permite uma avaliação mais eficiente da incerteza, a partir do levantamento detalhado de todos componentes de incerteza. O levantamento da contribuição relativa dos componentes de incerteza permite otimizar os custos e a incerteza da medição a partir de intervenções ao nível das componentes de incerteza minoritárias e maioritárias respetivamente (Burr et al., 2016).

O objetivo do presente trabalho é o de apresentar uma alternativa eletroquímica de baixo custo, simples e com qualidade adequada à determinação de ácido úrico em amostras de soro fisiológico e soro humano, sendo para tal assumido que a adequação da medição é avaliada a partir da comparação da incerteza da medição com uma incerteza alvo pré-definida. Para estimativa de incerteza será utilizada as Simulações de Monte Carlo em comparação com os métodos de Kragten e Lei da Propagação das Incertezas.

\section{MATERIAL E MÉTODOS}

\subsection{Construção dos Eletrodos}

Para a detecção de ácido úrico em amostras de soro fisiológico e humano foi desenvolvido um eletrodo de carbono em uma matriz de teflon. Foi construído o eletrodo conjugado, utilizando-se de um tubo de teflon, de diâmetro de $3,5 \mathrm{~cm}$, no qual foram feitos três furos, sendo que dois furos, com maior diâmetro foram utilizados para suportar o compósito de Cu-nanografite-lignina (eletrodo de trabalho) e o outro apenas para o eletrodo de nanografite (contra eletrodo). Um destes eletrodos foi utilizado como eletrodo de trabalho, área geométrica de $0,5 \mathrm{~cm}^{2}$, e o segundo preparado apenas com o compósito de nanografite foi utilizado como contra eletrodo de área geométrica de $0,78 \mathrm{~cm}^{2}$. O terceiro furo recebeu a quente e sob pressão o fio de prata pura de área geométrica de $0,20 \mathrm{~cm}^{2}$, sendo este empregado como eletrodo de referência. Os contatos elétricos externos foram feitos com fios de cobre.

O compósito, lignina-nanografite, foi preparado em diferentes proporções para a realização da busca da melhor relação entre as diferentes ligninas e o nanografite. Para a preparação do compósito as diferentes ligninas foram misturadas com nanografite, óleo mineral e hexano para a obtenção de uma mistura homogênea. O compósito permaneceu sobe agitação mecânica por 24 horas a temperatura ambiente para evaporação do solvente e garantir a homogeneidade. Após o estudo da proporção, foi escolhida a mistura composta por $25 \%$ de lignina, $60 \%$ de nanografite e $15 \%$ de óleo mineral.

Posteriormente, para a consecução do preparo do eletrodo de trabalho, sobre o compósito foi eletrodepositado cobre metálico. Para tal, após mergulhar-se o eletrodo conjugado em solução contendo íons $\mathrm{Cu}$ (II) em meio de eletrólito de suporte, foram realizados 4 ciclos, empregando-se a técnica de voltametria cíclica, no intervalo de potenciais: de $-1,0$ a $1,4 \mathrm{~V}$ vs. Ag, e velocidade de varredura de $300 \mathrm{mV} \mathrm{s}^{-1} \mathrm{em}$ $\mathrm{CuSO}_{4} 0,1 \mathrm{~mol} \mathrm{~L}^{-1}$.

\subsection{Validação dos Procedimentos}

A validação do procedimento de medição envolveu a avaliação dos pressupostos do modelo de regressão linear utilizado, a avaliação da incerteza de medição e a avaliação externa do modelo de medição a partir da análise de soros fisiológicos e dos soros humanos marcados pelo método padrão. O modelo de regressão utilizado para construir a curva de calibração é o modelo linear de regressão dos mínimos quadrados (MMQ), também conhecido como modelo linear paramétrico não ponderado (Miller et al., 2005).

A incerteza da medição foi baseada na abordagem "bottom-up" (Bettencourt da Silva, 2006), a partir da qual os componentes da incerteza foram combinados usando a lei de propagação da incerteza (LPI), método numérico de Kragten e Simulações de Monte Carlo (MCM). O método de Kragten foi utilizado para verificar os pressupostos de ambos os modelos de combinação de incerteza sobre a linearidade do modelo de equação dada à incerteza das quantidades de entrada (Kragten, 1994). O método de Kragten numérico foi particularmente 
útil para os pressupostos de avaliação dos modelos de regressão associados à qualidade dos calibradores. As simulações de Monte Carlo foram, ainda, utilizadas para avaliar as incertezas quando os pressupostos dos mínimos quadrados falham.

As curvas de calibração foram construídas, variando-se "sinal elétrico*volume total analito vs. massa de analito adicionado", $I_{t} * v_{t a} v s . m_{a}$. E para verificar a confiabilidade foi comparada a incerteza calculada experimentalmente com a incerteza alvo e realizado um teste de compatibilidade metrológica segundo guia EURACHEM/CITAC (Eurachem, 2012).

\subsection{Simulações de Monte Carlo e Pressupostos do MMQ}

O cálculo da estimativa da incerteza pode ser estimado com a LPI ou método numérico de Kragten utilizando o modelo de regressão dos mínimos quadrados, no entanto, é necessário seguir-se alguns pressupostos: 1homogeneidade de variância; 2- linearidade da resposta instrumental em função da concentração; e 3- irrelevância da incerteza associada ao teor relativo dos padrões de calibração, considerando-se a repetibilidade da resposta instrumental. Este último pressuposto refere-se ao preparo dos calibradores, os quais devem ser preparados corretamente.

Quando se dispõem de calibradores ruins torna-se difícil a aplicação da Lei da Propagação das Incertezas (LPI) ou Kragten para se estimar a incerteza da medição. Nesta situação, precisase de muita atenção, pois, poderá ocorrer de se subestimar as estimativas e os métodos de LPI ou Kragten falharem nos testes de compatibilidade. Em comparação com a LPI, as simulações de Monte Carlo (Herrador et al., 2005), são inerentes a preparação dos calibradores, podendo ser aplicada em todas as situações, mesmo na presença de calibradores ruins (Theodorou et al., 2011).

Para testar a veracidade deste pressuposto, preparou-se uma amostra fortificada de soro comercial contendo $3,0 \mathrm{mg} \mathrm{dL}^{-}$

${ }^{1}$ de $A U$ e foi realizada adições sucessivas de $100 \mu \mathrm{L}$ com uma pipeta graduada não calibrada e sem controle volumétrico. Em paralelo, realizou-se o mesmo experimento, ajustando os volumes adicionados por massa.

\section{RESULTADOS E DISCUSSÃO:}

\subsection{Comportamento eletroquímico do eletrodo Cu- nanografite-lignina}

Inicialmente, buscou-se investigar a atividade redox do ácido úrico, presente em solução aquosa, sobre a superfície do eletrodo de nanografite, na ausência e na presença de lignina. O comportamento eletroquímico de ácido úrico foi estudado em um intervalo de potencial, $(-1,0 \leq \mathrm{E} \leq 1,0) \vee v s$. Ag, empregando-se uma velocidade de varredura de potenciais de $300 \mathrm{mV}$ $\mathrm{s}^{-1} \mathrm{em} \mathrm{pH} 8$.

Foram investigadas duas situações: em ausência de ácido úrico; e em presença de ácido úrico $0,2 \mathrm{mmol} \mathrm{L}^{-1}$; e fazendo-se uso de quatro configurações diferentes de eletrodos: 1nanografite; 2- Cu-nanografite; 3- nanografitelignina; e 4- Cu-nanografite-liginina. Os eletrodos na presença de cobre ocorrem dois processos eletroquímicos (Figura 1), estando estes processos atribuídos as etapas de oxidação das espécies $\mathrm{Cu}(0)$ à $\mathrm{Cu}(\mathrm{I})$ (pico I e IV) e $\mathrm{Cu}(\mathrm{I})$ à $\mathrm{Cu}$ (II) (picos II e III). No entanto fica nítida a intensificação da intensidade de corrente de pico anódica de aproximadamente três vezes, para a preparação Cu-nanografite-lignina, em comparação a preparação nanografite-lignina, a qual de per si apresenta uma intensidade de corrente de pico superior a preparação anteriores.

Assim, a intensificação na corrente de pico para o eletrodo do compósito $\mathrm{Cu}$ nanografite-lignina, em presença da espécie eletroativa, ácido úrico, pode ser atribuído a esta espécie, possivelmente, devido à presença de um processo catalítico e, ou facilitado, pelo substrato e, especificamente, atribuído a presença do cobre metálico. Neste sentido, é importante notar-se que para os eletrodos preparados na ausência do cobre metálico verifica-se unicamente a presença de um par redox, sendo este processo atribuído a oxidação/redução da molécula de ácido úrico, porém, sem a mediação eletrônica do cobre. 


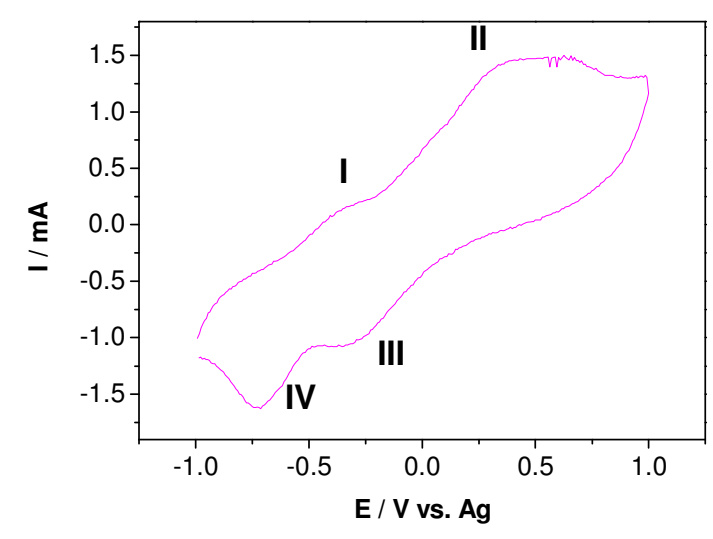

Figura 1: Voltamogramas cíclicos obtidos com eletrodo de Cu-nanografite-lignina em $\mathrm{KCl}$ 0,1 $\mathrm{mol} \mathrm{L}^{-1}(\mathrm{pH} 8) ; v=300 \mathrm{mV} \mathrm{s}^{-1} ; C_{A U}=2,0 \times 10^{-4} \mathrm{~mol}$ $L^{-1}$

\subsection{Construção dos Eletrodos}

Depois de estimada a incerteza do método colorimétrico de referência e do método eletroquímico alternativo, calculou-se as concentrações de ácido úrico nas amostras com os dois métodos, além da recuperação para as amostras de soro fisiológico. A Tabela 1 apresenta os resultados. A Tabela 1 também apresenta os resultados da avaliação da compatibilidade metrológica dos valores estimados e de referência utilizando a condição da Equação (1) (Eurachem, 2012). Se esta condição for válida, os valores estimados experimentalmente e de referência são metrologicamente compatíveis.

$$
\left|\gamma_{E}-\gamma_{R}\right| \leq t_{E}^{99 \%} \sqrt{u^{2}\left(\gamma_{E}\right)+u^{2}\left(\gamma_{R}\right)}
$$

No qual $\gamma_{E}$ e $\gamma_{R}$ são as concentrações de massa estimadas pelo procedimento eletroquímico e o valor de referência acrescido respectivamente, $\quad u\left(\gamma_{E}\right) e u\left(\gamma_{R}\right)$ são as incertezas padrões do método eletroquímico e do método de referência e $t_{E}^{99 \%}$ o $t$ de student para $99 \%$ de confiança e graus de liberdade referente a $u_{E}$. Os graus de liberdade da diferença $\left(\gamma_{E}-\gamma_{K}\right)$ são equivalentes aos de $\gamma_{E}$ uma vez que os graus de liberdade de $\gamma_{R}$ são esperados ser maiores. Os graus de liberdade do resultado da combinação linear de duas variáveis são denominados pelo número de graus de liberdade do componente com menos graus de liberdade.

A compatibilidade também pode ser testada verificando se o valor absoluto estimado da diferença $\left(\left|\gamma_{E}-\gamma_{R}\right|\right)$ com a respectiva incerteza expandida, isto é, o termo no lado direito de "s" da Equação (1)) inclui o valor zero.

Os valores estimados pelo procedimento eletroquímico são metrologicamente equivalentes às concentrações estimadas pelo procedimento de adição e recuperação nas amostras de soro fisiológico, demonstrando a confiabilidade das medidas eletroquímicas incluindo a avaliação da incerteza.

A Tabela 1 também apresenta os resultados da avaliação da compatibilidade metrológica de medições de referência e alternativas usando a condição da Equação (1).

Tabela 1: Resultados da determinação de ácido úrico em soro fisiológico e soro humano $e$ respectivos valores de referência.

\begin{tabular}{lcc}
\hline & $\begin{array}{c}\text { Conc, método } \\
\text { eletroquímico } \\
\left(\mathbf{m g ~ d L}^{-1}\right)\end{array}$ & $\begin{array}{c}\text { Conc, método } \\
\text { colorimétrico } \\
\left(\mathbf{m g ~ d L}^{-1}\right)^{*}\end{array}$ \\
\hline Soro 01 & $1,03 \pm 0,27$ & $1,00 \pm 0,02$ \\
Soro 02 & $3,03 \pm 0,32$ & $3,02 \pm 0,06$ \\
Soro 03 & $5,06 \pm 0,38$ & $5,04 \pm 0,09$ \\
Soro 04 & $7,01 \pm 0,49$ & $7,06 \pm 0,13$ \\
Soro 05 & $8,93 \pm 0,56$ & $9,07 \pm 0,17$ \\
\hline SoroH 01 & $3,83 \pm 0,50$ & $4,10 \pm 0,12$ \\
SoroH 02 & $5,92 \pm 0,64$ & $6,20 \pm 0,12$ \\
\hline
\end{tabular}

Como mostrado na Tabela 1, os dois pares de resultados são metrologicamente compatíveis, uma vez que o valor absoluto de sua diferença para todas as amostras é menor do que a incerteza expandida da diferença, demonstrando a equivalência metrológica dos resultados produzidos por ambos os procedimentos.

Portanto, pode-se concluir que o procedimento eletroquímico alternativo é uma alternativa válida ao ácido úrico estimado em amostras de soro. Os resultados da Tabela 1 também permitem concluir que ambos os têm valores normais de ácido úrico, uma vez que estes estão posicionados dentro do intervalo de 2 $\mathrm{mg} \mathrm{dL}^{-1}-8 \mathrm{mg} \mathrm{dL}^{-1}$.

\subsection{Comparação Monte Carlo e sua utilização em amostras para as quais os calibradores falham}

Nas amostras estudadas foi utilizado o método de Kragten e a LPI para o cálculo da 
estimativa de incerteza, mas estes métodos somente se aplicam se os calibradores forem devidamente preparados.

Caso os calibradores não forem devidamente preparados, a estimativa de incerteza é subestimada, encontrando-se valores abaixo do "valor real", e com isso obtêm-se resultados errôneos. Com valores subestimados de estimativa de incerteza e valores de concentrações longe dos ideias, a chance de não compatibilidade (equação 1) é demasiadamente alta. Mas quando se usa as simulações de Monte-Carlo, não se tem a necessidade de um controle rígido dos calibradores, pois quando se simula uma quantidade extremamente grande de experimentos, é considerada a contribuição mais próxima do real de cada fonte de incerteza.

Para comprovar tal fato, foi conduzido um experimento, no qual a célula eletroquímica foi inserida dentro de uma balança analítica e feita as adições de padrão de $100 \mu \mathrm{L}$ com uma pipeta graduada, sem nenhum controle analítico rígido e construída duas curvas de calibração, uma de "sinal*volume total vs. massa de analito adicionado" $\left(I_{t} * v_{t a}\right.$ vs. $\left.m_{a}\right)$ e outra de "sinal"massa total vs. massa de analito adicionado" $\left(I_{t} * m_{t a}\right.$ vs. $\left.m_{a}\right)$, no qual o primeiro assume-se que está adicionando $100 \mu \mathrm{L}$ do padrão e na segunda assume-se a pesagem equivalente a estes $100 \mu \mathrm{L}$ adicionados do padrão. Em ambos os casos foi analisado a qualidade dos calibradores e depois estimada as incertezas utilizando a LPI e as simulações de Monte-Carlo e feito o teste de compatibilidade (equação 1). A amostra analisada foi de soro fisiológico contendo $3,0 \mathrm{mg} \mathrm{dL}^{-1}$ de ácido úrico.

Primeiramente foi analisada a qualidade dos calibradores por volume, no qual o valor de $u\left(R_{a_{i j j}}\right) / R_{a_{i j j}}$ foi maior que um quinto de $u\left(R_{v I_{i j}}\right) / R_{v I_{i j}}$ demonstrando que utilizando uma pipeta sem controle analítico gera calibradores ruins. Mas quando se usa as mesmas equações para testar a qualidade dos calibradores considerando-se a massa total pesada, o valor de $\left(R_{a_{i j}}\right) / R_{a_{i j j}}$ é menor que um quinto de $u\left(R_{w I_{i, j}}\right) / R_{w I_{i, j}}$, demonstrando que por pesagem a qualidade do calibrador é boa, mesmo utilizando-se instrumentação sem calibração prévia. Esta diferença nos resultados se deve ao fato de que a incerteza da pesagem é inferior a incerteza do volume medido, principalmente quando se trabalha na escala de micro volumes.

Uma vez analisado os calibradores, foi estimada cada fonte de incerteza pela LPI e pelo MCM. A Tabela 2 apresenta os resultados e as respectivas incertezas para ambas abordagens de cálculo.

Para as simulações de Monte Carlo, o cálculo de estimativa de incerteza foi procedido criando-se uma folha de cálculo e aplicando-se algoritmos específicos para cada fonte de incerteza (Cox et al., 2006). O princípio baseia-se em inserir cada variável e suas respectivas fontes de incerteza, e em seguida realizar simulações de modo aleatório de acordo com o tipo de distribuição que se aplica para cada uma dessas fontes. A partir destas simulações, podese construir um gráfico de distribuição normal, conforme apresentado na Figura 2, e a partir deste gráfico encontrar a concentração da amostra e a respectiva incerteza para $95 \%$ ou $99 \%$ de confiança.

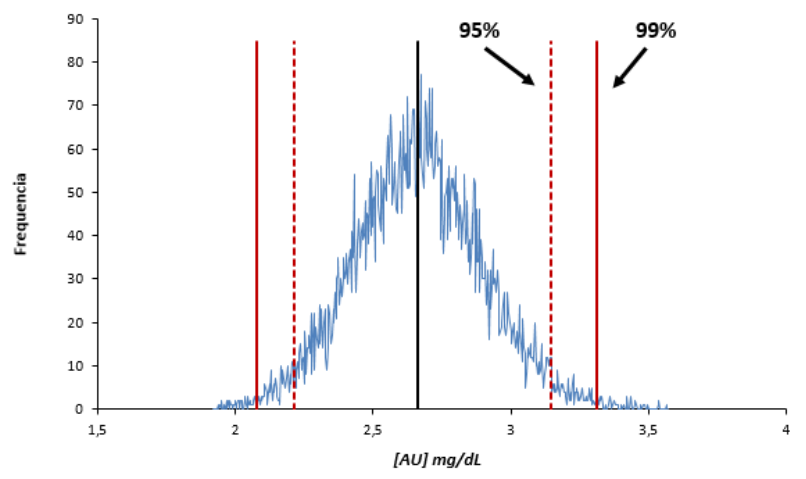

Figura 2: Distribuição normal para as simulações de Monte Carlo na amostra de soro fisiológico contendo $A U$ nas medições considerando sinal ${ }^{*}$ volume total vs. massa de $A U$ adicionado

A partir da Figura 2, pode-se observar que o ponto central é a média das simulações e as linhas nas extremidades representam a incerteza expandida da amostra $\left(U\left(\gamma_{S}\right)\right)$ para $95 \%$ e $99 \%$ de confiança. Com os resultados estimados pela LPI e MCM, construiu-se a Tabela 2 para comparação das duas abordagens.

Como pode ser evidenciada pela Tabela 2, a incerteza do fator de diluição para o procedimento de pesagem é menor que comparado com o de volume, devido à incerteza de pesagem ser menor que quando adicionado pequenos volumes. As recuperações nas medidas de massa são bem maiores do que as de volume e todas passam pelo $100 \%$. 
No teste de compatibilidade realizado pelo procedimento de pesagem, têm-se ambos compatíveis e suas incertezas menores do que comparado com os procedimentos volumétricos, inclusive comparando-se com as amostras estudadas na sessão anterior.

Quando se utiliza o procedimento volumétrico sem o controle analítico, observa-se que a recuperação é bem menor que por massa, além de não ser compatível quando se utiliza a abordagem da LPI para estimativa de incerteza. Esse fato se deve que a LPI para ser estimada corretamente é necessário ter um controle rígido no preparo dos calibradores, pois senão têm-se incertezas subestimadas, como pode ser evidenciado a partir da Tabela 2, no qual a incerteza por pesagem apresentou um valor maior do que a incerteza por volume.

No entanto, ao se aplicar as simulações de Monte Carlo, observa-se que ocorre compatibilidade, mesmo a recuperação sendo pequena, pois a estimativa de incerteza nesta abordagem não é subestimada, tendo valores bem maiores que a LPI. A vantagem da aplicação das simulações de Monte Carlo é que a incerteza tende a ser estimada corretamente, pois esta abordagem não necessita de controle analítico rígido.

Ela é bem aplicada para sistemas no qual se tem adições de pequenos volumes ou que não se prepara as soluções por pesagem. A sua desvantagem são os recursos computacionais de algoritmos que são necessários para sua estimativa (Sega et al., 2016).

\section{CONCLUSÕES}

O eletrodo de nanografite modificado com lignina e cobre eletrodepositado foi muito eficiente para a preparação de um sensor eletroquímico para a determinação de ácido úrico em soro humano. A superfície modificada pela eletrodeposição de cobre revelou a presença de um mecanismo do tipo ECE para a oxidação da $\mathrm{AU}$.

Uma vez que a resposta do eletrodo varia com a matriz da amostra e estão disponíveis pequenos volumes de soro para análise, a calibração do sensor foi realizada pelo método de adição de padrão com adições de padrão cumulativo. Na calibração, a "corrente de oxidação da célula eletroquímica", $I_{\mathrm{ta}}$, vezes o "volume total da solução", $v_{t a},\left(I_{t a} *_{t a}\right)$ é proporcional à massa de AU adicionada $\left(m_{a}\right)$. A curva de calibração foi utilizada para extrapolar a massa de AU na amostra e esta massa foi convertida em concentração de AU tomando a alíquota volumétrica da amostra.

O sinal instrumental é homocedástico e varia linearidade com a quantidade $A U$, e a metodologia, desenvolvida para realizar adições cumulativas, não afetou a regressão estatística na calibração.

Todas as medições eletroquímicas apresentam incertezas de medição baixas, uma vez que não foram comprovadas serem superiores a incerteza alvo, 0,56 $\mathrm{mg} \mathrm{dL}^{-1}$.

A qualidade da medição realizada foi avaliada a partir da análise de amostras de soro fisiológico e de humanos cujos valores de referência foram estimado pelo processo de adição/recuperação e por um método padrão independente, respectivamente. Desta forma, sendo as medidas metrologicamente compatíveis com as medidas de referência, o procedimento de medição, incluindo a sua estimativa da incerteza, é fiável.

Devido à sua estabilidade, seletividade, simplicidade de trabalho e baixo custo de construção, o sensor construído trata-se de alternativa promissora ao procedimento de referência.

\section{REFERÊNCIAS:}

1. Benini, S., Cianci, M., Mazzei, L. J Biol Inorg Chem, 2014, 19, 1243.

2. Bettencourt da Silva, R.J.N. Talanta, 2016, 148, 177.

3. Bettencourt da Silva, R.J.N., Santos, J.R., Camões, M.F.G.F. Accred. Qual. Assur., 2006, 664.

4. Burr, T., Croft, S., Krieger, T., Martin, K., Norman, C., Walsh, S. Appl. Radiat. Isot, 2016, 108, 49.

5. Cox, M.G., Siebert, B.R.L. Metrologia, 2006, 43, S178.

6. Eurachem/CITAC: Quantifying uncertainty in analytical measurement, in: S.L. R., Ellison, A., Williams (Eds.), third ed., Eurachem, 2012.

7. Filik, H., Avan, A.A., Aydar, S., Arpacı, R.B. Int. J. Electrochem. Sci., 2014, 9, 2775.

8. Goncalves, D.A., Jones, B.T., Donati, G.L. Microchem J, 2016, 124, 155. 
9. Guo, X.M., Guo, B., Li, C., Wang, Y.L. J. Electroanal. Chem, 2016, 783, 8.

10. Herrador, M.A., Asuero, A.G., González, A.G. Chemom. Intell. Lab. Syst, 2005, 79, 115.

11. Jin, D., Seo, M., Huy, B., Pham, Q., Conte, M. L., Thangadurai, D., Lee, Y. Biosens. Bioelectron, 2016, 77, 359.

12. Joint Committee for Guides in Metrology, International Vocabulary of MetrologyBasic and General Concepts and Associated Terms (VIM), JCGM 200, BIPM, 2012.

13. Kageyama, N. Clin Chim Acta, 1971, 31, 421.

14. Khajehsharifi, H., Pourbasheer, E., Tavallali, H., Sarvi, S., Sadeghi, M., Arab. J. Chem, 2017, 10, S3451.

15. Kragten, J. Analyst, 1994, 119, 2161.

16. Martin, C.S., Dadamos, T.R.L., Teixeira, M.F.S. Sens Actuators B Chem, 2012, 175, 111.
17. Miller, J., Miller, J. Statistics and Chemometrics for Analytical Chemistry, 5th ed., UK: Pearson, 2005.

18. Moriwaki, Y. J Bioequiv Availab, 2014, 6 10.

19. Rho, Y.H., Zhu, Y., Choi, H.K. Semin. Nephrol., 2011, 31, 410-419.

20. Sega, M., Pennecchi, F., Rinaldi, S., Rolle, F., Anal. Chim. Acta, 2016, 920, 10.

21. Steliopoulos, P. MethodsX, 2015, 2, 353.

22. Theodorou, D., Meligotsidou, L., Karavoltsos, S., Burnetas, S., Dassenakis, M., Scoullos, M., Talanta, 2011, 83, 1568.

23. Uzun, D., Gündüzalp, A.B., Hasdemir, E. J. Electroanal. Chem, 2015, 747, 68.

24. Wu, A.H., Gladden, J.D., Ahmed, M., Ahmed, A., Filippatos, G. Int. J. Cardiol, 2016, 213, 4.

25. Zuo, T., Jiang, L., Mao, S., Liu, X., Yin, X., Guo, L. Int. J. Cardiol, 2016, 224, 286.

Tabela 2: Resultados comparativos de estimativa de incerteza por medições em massa e volume, utilizando as abordagens da LPI e MCM

\begin{tabular}{ccccc} 
& \multicolumn{2}{c}{ Massa } & \multicolumn{2}{c}{ Volume } \\
\cline { 2 - 5 } & LPI & MCM & LPI & MCM \\
\cline { 2 - 5 } $\boldsymbol{u}^{\prime}\left(\boldsymbol{d}_{\mathbf{1}}\right)(\%)^{*}$ & 0,14 & - & 0,23 & - \\
$\boldsymbol{u}_{\mathrm{e} X t}(\%)^{*}$ & 0,59 & - & 0,46 & - \\
Concentração método eletroquímico $\left(\mathbf{m g ~ d L}^{-1}\right)$ & $2,93 \pm 0,23$ & $2,90 \pm 0,20$ & $2,68 \pm 0,19$ & $2,67 \pm 0,47$ \\
Concentração método colorimétrico $\left(\mathbf{m g ~ d L}^{-1}\right)$ & $3,02 \pm 0,01$ & $3,02 \pm 0,01$ & $3,02 \pm 0,03$ & $3,02 \pm 0,03$ \\
Recuperação (\%) & $97 \pm 15$ & $97 \pm 13$ & $89 \pm 13$ & $88 \pm 30$ \\
Teste de Compatibilidade & $0,09 \pm 0,23$ & $0,12 \pm 0,40$ & $0,33 \pm 0,19$ & $0,35 \pm 0,94$ \\
\hline
\end{tabular}

PERIÓDICO TCHÊ QUÍMICA • www.periodico.tchequimica.com • Vol. 15 N. 29

- ISSN 1806-0374 (impresso) • ISSN 1806-9827 (CD-ROM)・ISSN 2179-0302 (meio eletrônico) (C) 2018. Porto Alegre, RS. Brasil 